Document downloaded from:

http://hdl.handle.net/10251/123285

This paper must be cited as:

Ruiz, A.; Panach Navarrete, Jl.; Pastor López, O.; Giraldo-Velásquez, FD.; Arciniegas, JL.; Giraldo, WJ. (2018). Designing the Didactic Strategy Modeling Language (DSML) From PoN: An Activity Oriented EML Proposal. IEEE-RITA: Latin-American Learning Technologies Journal. 13(4):136-143. https://doi.org/10.1109/RITA.2018.2879262

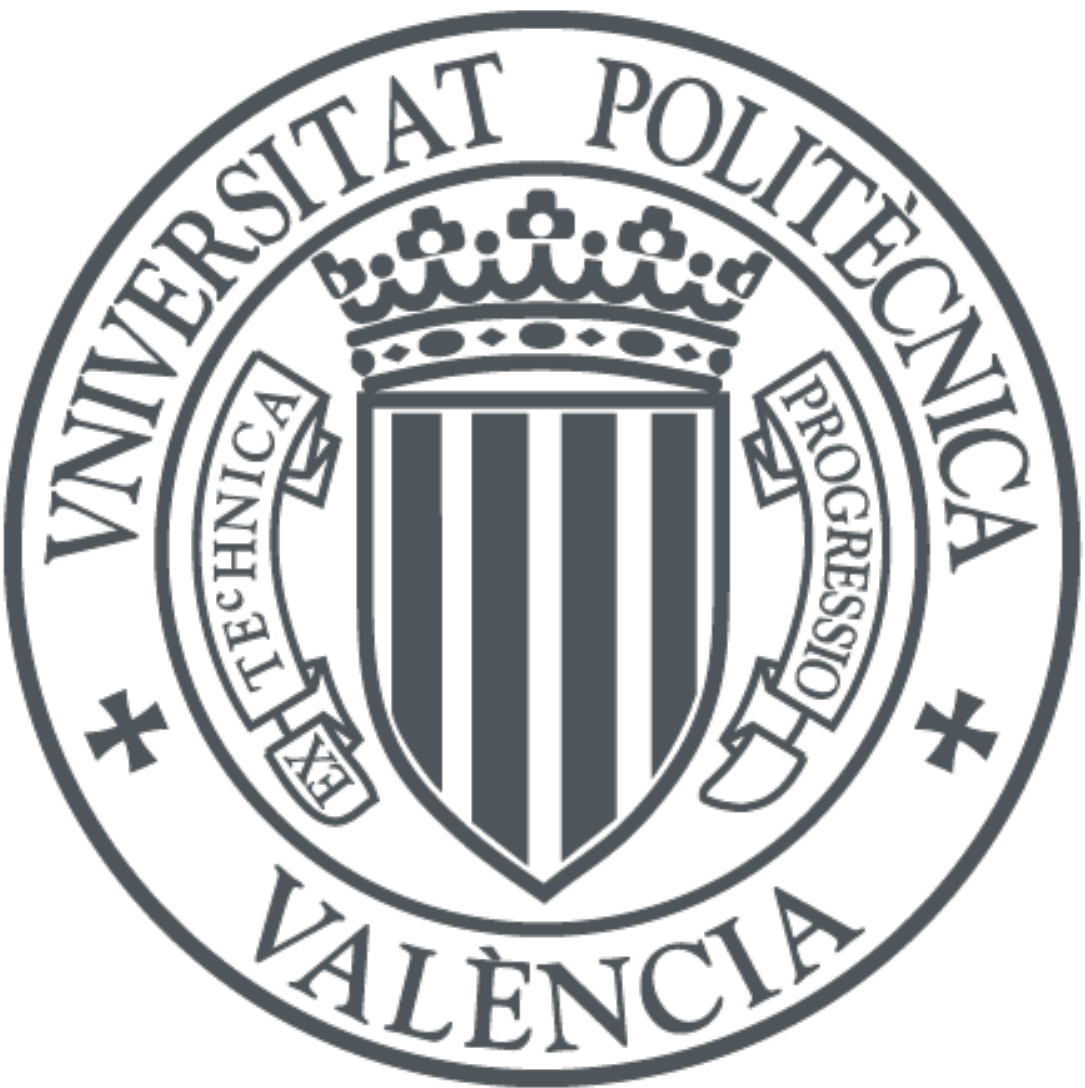

The final publication is available at

http://doi.org/10.1109/RITA.2018.2879262

Copyright Institute of Electrical and Electronics Engineers

Additional Information 


\title{
Designing the Didactic Strategy Modeling Language (DSML): An Activity Oriented EML Proposal
}

\author{
A. Ruiz, J. I. Panach, O. Pastor, F. D. Giraldo, J. L. Arciniegas y W. J. Giraldo
}

\begin{abstract}
This article presents the design of Didactic Strategy Modeling Language (DSML), according to the principles of Physics of Notations (PoN). DSML is a visual and activity-oriented language for learning design whose main characteristic lies in the representation of the different activities according to the nature of the task. The language requirements are established based on the exploration of strengths and weaknesses of existing Educational Modeling Languages (EML) proposals. Once the language is designed, a blind interpretation study is conducted to validate the semantic transparency of the learning activity iconography. The results of the study allow to refine icons. Also, an authoring tool for DSML is presented, which is integrated to an LMS. A Model Driven course is designed as a DSML pre-validation.
\end{abstract}

Keywords-sequences of activities, DSML, didactic strategy, educational modeling languages, instructional design

\section{INTRODUCTION}

$\mathrm{L}$ A estrategia didáctica, definida como el procedimiento con el que el profesor pretende facilitar los aprendizajes en los estudiantes a través de actividades que contemplan la interacción de los alumnos con determinados contenidos [1], es uno de los elementos más importantes del proceso de enseñanza-aprendizaje. De acuerdo a la definición, la estrategia didáctica se compone de actividades didácticas que al ser ejecutadas por el estudiante cobran su valor de actividades de aprendizaje. Es de esperar que, de acuerdo a los aprendizajes previos, capacidades y formas de aprender de los estudiantes, el docente planee una serie de estrategias didácticas que se adapten a las necesidades de grupos de estudiantes de características similares. Aunque la planeación y ejecución de estrategias didácticas personalizadas pueden ser realizadas por el docente sin apoyo de tecnología, actualmente las plataformas virtuales juegan un papel importante a la hora de facilitarle esta labor.

En aras de poder diseñar y ejecutar estrategias didácticas en entornos computacionales, más ampliamente conocidas en el argot de e-learning como "secuencias de actividades"[2], han surgido diferentes propuestas basadas en el modelado. En particular, los Lenguajes de Modelado Educativo (EMLs: Educational Modeling Languages) [3] han sido propuestos para permitir el modelado computacional de unidades didácticas, de forma que se faciliten los procesos de diseño, comunicación y desarrollo de las mismas. Actualmente, existe el lenguaje estándar de facto IMS-LD [4] (Instructional Management Systems Learning Design) cuya finalidad principal es la creación de modelos de unidades didácticas computacionales, de forma que el desarrollo de las mismas pueda ser controlado y soportando a través de sistemas TICs. Este estándar, sin embargo, no plantea la representación gráfica de las secuencias de actividades [5] cómo sí evidencian otras propuestas como PoML[5], CoUML[6], E²EM[7], CoLeML[8] o MISA/MOT+[9, 10], entre otras.

No obstante, las propuestas en mención tienen carencias en relación a la calidad de la notación y otros aspectos importantes a considerar en un EML, tal como se evidencia en la sección de trabajos relacionados que más adelante se presenta en este artículo.

Como alternativa de solución a las carencias encontradas, proponemos en este artículo el lenguaje para el modelado de estrategias didácticas DSML (Didactic Strategy Model Language). DSML es un lenguaje gráfico para el modelado de estrategias didácticas que puede ser utilizado en entornos computacionales. Una de las características más relevantes de este lenguaje es la representación de las diferentes actividades didácticas de acuerdo a la taxonomía de Conole [11], en donde se identifican diferentes tipos de actividad de acuerdo a la naturaleza de la tarea. La representación de los diferentes tipos de actividad en el lenguaje permite identificar fácilmente patrones de enseñanza que pueda dar a lugar a acciones de mejora o correctivas según sea el caso. Dependiendo de la naturaleza de cada tarea se identifica el conjunto de herramientas y recursos que los estudiantes pueden usar para completar la tarea. DSML se construye a partir del estudio de propuestas existentes y de la aplicación sistemática de los principios principios de la Física de las Notaciones (Physics of Notations - PoN) de D.L Moody [12]. La aplicación de estos principios provee una base científica para la comparación, evaluación, mejora y construcción de notaciones visuales. Finalmente, se crea una herramienta de autoría para el lenguaje independiente del Sistema de Gestión de Aprendizaje (Learning Management System - LMS) [13]. Como ejemplo de integración en un LMS especifico, la herramienta se integrada a la plataforma Moodle bajo la aplicación del método expuesto en [14]. 
Para este fin, la sección 2 expone algunas de las herramientas EML analizadas, analizando en detalle sus ventajas y desventajas, a partir de las cuales se definieron los requisitos del lenguaje propuesto. La sección 3 presenta los conceptos que guían la investigación con relación a como se concibe el modelo de estrategia didáctica. La sección 5 presenta en detalle el diseño del lenguaje para el modelado de estrategias didácticas DSML aplicando PoN. La sección 6 presenta la herramienta de autoría diseñada para DSML y su integración en un LMS, específicamente, en la plataforma Moodle. Finalmente, las conclusiones y trabajos futuros se presentan en la sección 7.

\section{TRABAJOS RELACIONADOS}

Desde el punto de vista pedagógico, un EML es una notación que docentes e instructores pueden usar para formalizar los diseños del aprendizaje que tienen en mente. En contraposición al lenguaje natural, la aproximación formal permite el procesamiento automático por sistemas computacionales. Desde el punto de vista técnico, el EML puede también ser visto como un lenguaje de escritura o scripting language para plataformas de aprendizaje o LMSS que permite la configuración de experiencias de aprendizaje sobre estos sistemas [15]. En la literatura se evidencian diferentes EMLs orientados a modelar unidades de aprendizaje, algunos enmarcados en la perspectiva pedagógica y otros en la perspectiva técnica o formal. Esta sección presenta algunos de los EMLs representativos que fueron explorados con el fin de analizar su viabilidad en entornos reales. Para cada lenguaje se resaltan sus ventajas y desventajas de acuerdo con estudios encontrados en la literatura y, seguidamente, una tabla resumen clasifica las propuestas de acuerdo con los aspectos importantes que caracterizan a los EML. Finalmente, se enumeran los requisitos para el nuevo lenguaje que se diseña para solucionar las desventajas que se establecen como resultado de la exploración.

$\mathbf{E}^{2} \mathbf{M L}$ [16]: es un lenguaje con un conjunto de tipos de diagramas que cubren dos capas de detalle (sobre la vista y de la acción) y dos perspectivas (temporal y estructural) diferentes. Los objetivos de aprendizaje, los requisitos y el diseño de las actividades de enseñanza y aprendizaje pueden ser modelados. $\mathrm{E}^{2} \mathrm{ML}$ permite mejorar la calidad de la instrucción y suavizar el proceso de diseño. Sin embargo, en [17] se evidencian desventajas como: algunos usuarios lo estiman muy complicado y con demasiados elementos, no se percibe ninguna recompensa en el esfuerzo requerido para especificar la documentación y algunos de los diagramas tienen falencias a nivel de su efectividad cognitiva, especialmente el diagrama de objetivos.

IMS-LD [18]: IMS Learning Design está basado en el lenguaje de modelado educacional (Educational Modeling Language - EML) y define una técnica de modelado y enlaces mediante XML para describir roles y secuencias de actividades dentro de un ambiente de objetos y servicios de aprendizaje. Las propiedades, condiciones y notificaciones se proporcionan en otros niveles. Su objetivo principal es proporcionar un medio para el intercambio y la ejecución (semiautomática) de los diseños de aprendizaje [19]. IMS-LD se basa en un modelo conceptual bien fundamentado y documentado que define el vocabulario y las relaciones funcionales entre los conceptos del diseño de aprendizaje [20]. Además, permite especificar, formalizar e implementar procesos de aprendizaje [21]. No obstante, se identifican desventajas como: deficiencias de expresividad [22] y la edición de un curso de acuerdo con el modelo completo de IMS LD es engorroso [23] y complejo para el profesor promedio [24].

PoEML [22]: el lenguaje de modelado educativo orientado a perspectiva (Perspective-oriented Educational Modeling Language - PoEML) integra conceptos del modelado de flujo de trabajo y groupware. Se centra en la separación de 13 perspectivas diferentes sobre diseños educativos (estructural, funcional, participantes, entorno, datos, herramientas, organizacional, orden, temporal, autorización, awareness, interacción, causal). De esta manera, las personas involucradas en el diseño y el desarrollo de unidades didácticas pueden realizar su trabajo centrando la atención en una perspectiva cada vez [5]. Además, PoEML puede ser usado para modelar escenarios educativos sobre diferentes niveles de agregación (lecciones, currículos) y ofrece un conjunto de patrones para modelar en cada una de las perspectivas [19]. Sin embargo, se evidencian deficiencias en la notación visual que ofrecen los símbolos del lenguaje como: existen demasiados tipos de diagramas y una gran cantidad de símbolos, no hay suficientes diferencias visuales para distinguir uno símbolo de otro y algunos de los símbolos no dan a entender su significado [25].

CoUML [6]: este lenguaje, basado en UML, expone un sistema de notación formal que permite a) modelar conceptos estructurales como los documentos, las metas y los roles involucrados; y, b) el modelado de las actividades realizadas por los roles en el entorno objetivo, incorporando objetos relevantes de los modelos estructurales (e.g. los documentos utilizados en las actividades o producidos por ellas o los objetivos alcanzados por las actividades) [26]. Al ser una extensión de UML tiene la ventaja que puede ser llevado fácilmente a la implementación [27]. Igualmente, el lenguaje reporta buenos resultados a nivel de efectividad cognitiva [25]. Sin embargo, y como desventaja, cabe mencionar que, a pesar de que sus autores afirman que CoUML es un lenguaje pedagógicamente neutral, su uso puede ser difícil debido a que las personas son reacias a usar UML por estar estrechamente relacionado con las ciencias de la computación [27].

ColeML [8]: hace posible que los diseñadores de aprendizaje diseñen modelos que apoyen a estudiantes y profesores trabajando en proyectos, ayudándoles a estructurar los procesos y a organizar la colaboración. El desarrollo de ColeML se ha inspirado en el trabajo en el área de modelado del flujo de trabajo y proceso de negocio. ColeML se basa en WorkWare de Jørgensen y sus principios [28]. El lenguaje maneja pocos símbolos, lo cual lo hace fácil de aprender, sin embargo, desde el punto de vista de los autores, dichos símbolos no reflejan su significado. No se evidencian estudios realizados sobre el lenguaje. 
Como resumen, y usando el esquema de clasificación de lenguajes visuales para diseño instruccional introducido por Botturi et al. [19], la Tabla I muestra la clasificación de las propuestas EML anteriormente analizadas. El atributo "Usuario" es adicionado a la clasificación, el cual denota la audiencia que es más adecuada para usar el lenguaje. La clasificación propone los siguientes clasificadores: estraficación $(E s)$, formalización $(F o)$, Elaboración $(E l)$, perspectiva $(\mathrm{Pe})$, creatividad $(\mathrm{Cr})$. El atributo Usuario $(U s)$ es adicionado a la clasificación, el cual denota la audiencia que es más adecuada para usar el lenguaje.

TABLA I

CLASIFICACIÓN DE EMLS DE ACUERDO LOS ATRIBUTOS DEL ESQUEMA DE BOTTURI [19] + EL CLASIFICADOR "USUARIO"

\begin{tabular}{ccccc}
\hline \hline & E $^{2} \mathbf{E M}$ & PoEML & IMS-LD & CoUML \\
\hline$E s$ & Plano & Capas & Capas & Capas \\
$F o$ & SemiFormal & Formal & Formal & SemiFormal \\
$E l$ & Conceptual & Implementación & Especificación & Especificación \\
$\mathrm{Pe}$ & Múltiple & Múltiple & Simple & Múltiple \\
$\mathrm{Cr}$ & Generativo & Finalista & Finalista & Finalista \\
$\mathrm{Us}$ & Diseñador & Diseñador & Desarrollador & Diseñador \\
\hline
\end{tabular}

\section{A. Requisitos para un nuevo lenguaje EML}

La exploración de las anteriores propuestas representativas permitió establecer los requisitos necesarios para la nueva propuesta de lenguaje a diseñar. A continuación, se listan tales requisitos:

(i) R1: El modelo conceptual que soporta el lenguaje debe partir del contexto educativo.

(ii) R2: El lenguaje debe permitir crear unidades de estudio que incluyan las actividades a realizar por el estudiante (que hacer), las personas involucradas en la actividad (por quien), y el entorno donde las actividades se realizarán (con qué).

(iii) R3: El lenguaje debe ser fácil de usar y de aprender debido a que la audiencia objetivo son los profesores en general.

(iv) R4: La notación del lenguaje se debe caracterizar por tener altos niveles de efectividad cognitiva de acuerdo a los principios establecidos por Moody [12].

(v) R5: El lenguaje debe ser visual con el fin de que facilite la comunicación y comprensión de la realidad modelada.

(vi) R6: Debe ser posible su procesamiento computacional.

\section{MODELO CONCEPTUAL DE LA ESTRATEGIA DIDÁCTICA}

Esta sección presenta los conceptos que guían la investigación con relación a cómo se concibe el modelo de estrategia didáctica. Con el fin de dar cumplimiento al requisito $\mathbf{R} 1$, el modelo de estrategia didáctica tiene sus raíces en el estudio de los modelos conceptuales del acto didáctico. Aunque la mayoría de los conceptos expuestos corresponden al modelo propuesto por Marquès [1], estos comparten elementos comunes a otras propuestas presentadas por los autores Meneses [29], Heinemann citado en [30], Rodríguez [31], González [32], Gimeno [33] y Ferrandez [34]. Como resultado del análisis de las diferentes propuestas del acto didáctico y de la naturaleza de las actividades de aprendizaje [11], en la Fig. 1 se presenta el modelo conceptual (metamodelo) de la estrategia didáctica. Los lectores deben considerar la Fig. 1 como una parte de todo el meta-modelo. Solo los conceptos y las relaciones estructurales son mostrados de acuerdo con los intereses de este artículo. A continuación, se presentan los conceptos que fueron pieza clave en la construcción del lenguaje para el modelado de la estrategia didáctica propuesta, los cuales se resaltan en negrita. Al lado de cada concepto se especifica entre paréntesis el constructor al que corresponde en el meta-modelo.

Según Marquès [1], el acto didáctico define la actuación del profesor (Staff) para facilitar los aprendizajes de los estudiantes (Student) y su naturaleza es esencialmente comunicativa. De acuerdo con el autor, la enseñanza se concreta en actos didácticos mediante los cuales el profesor o formador propone múltiples actividades a los estudiantes para facilitar los aprendizajes deseados. Estas actividades, conocidas como actividades de aprendizaje (LearningActivity), son adaptadas a las características de los estudiantes, a los recursos disponibles (Resource) y a los contenidos objeto de estudio. Sin embargo, el proceso de enseñanza no solo se compone de actividades de aprendizaje sino también de actividades de soporte (SupportActivity) [4], las cuales usualmente son realizadas por el profesor para soportar a los estudiantes. No obstante, en algunos modelos pedagógicos algunos estudiantes pueden apoyar a otros estudiantes (peer to peer teaching). Vemos de esta manera como diferentes actores o roles (Role) tienen su parte en la actividad, en donde un solo estudiante (Student), un grupo de estudiantes (Group), la clase (Class) y/o el personal a cargo (Staff) pueden estar involucrados. Usualmente, el personal a cargo es un profesor, pero también pueden haber auxiliares o colaboradores. Por otra parte, las actividades pueden realizarse en diferentes modos de presencialidad (PresenceMode): presencial (Face-to-face), combinado (Blended, apoyado en TICs) y online (WebBased, basadas completamente en la web).

Marquès plantea que la eficacia de los recursos didácticos dependerá en gran medida de la manera en la que el profesor oriente su uso en el marco de la estrategia didáctica (DidacticStrategy) que está utilizando. Una estrategia es, en un sentido estricto, un procedimiento organizado, formalizado y orientado a la obtención de una meta claramente establecida. De esta manera, la estrategia didáctica es el procedimiento con el que el profesor pretende facilitar los aprendizajes en los estudiantes a través de actividades que contemplan la interacción de los alumnos con determinados contenidos. Igualmente, la estrategia didáctica debe adaptarse a las necesidades de los estudiantes. Para ello, el docente debe establecer puntos de control (ControlPoint) que permitan evaluar el progreso del estudiante y de esta forma plantear diferentes flujos (OrderFlow y FlowControl) de actividades de acuerdo con el resultado de la evaluación. Algunos eventos (Event) también pueden establecerse en ciertos puntos del procedimiento, como, por ejemplo, el profesor desea ser notificado (notification) cuando el estudiante haya finalizado cierta actividad o desea que el estudiante no realice ninguna actividad hasta que tenga la retroalimentación de algún entregable, para lo cual establece un tiempo de espera (Wait). 
Por otra parte, Conole [11] propone una taxonomía que define los componentes que integran una actividad de aprendizaje. Uno de los aspectos más útiles de la taxonomía es la descripción detallada de la naturaleza de la tarea que los estudiantes realizarán como parte de la actividad de aprendizaje para alcanzar los objetivos deseados. Esta taxonomía es enriquecida por Marcelo [35], el cual incluye el tipo de actividad "evaluación" que no está presente en la versión original de la taxonomía. De esta forma, los tipos de actividades (Activity) de acuerdo con la naturaleza de la tarea pueden ser: asimilativas (Assimilative), de gestión de información (InformationHandling), de aplicación (Adaptive), comunicativas (Communicative), productivas (Productive), experienciales (Experiential) y evaluativas (Evaluative). Una subcategorización de estos tipos de actividad es propuesta por los autores a partir del análisis de las actividades didácticas recogidas en el estudio de Marcelo [35].

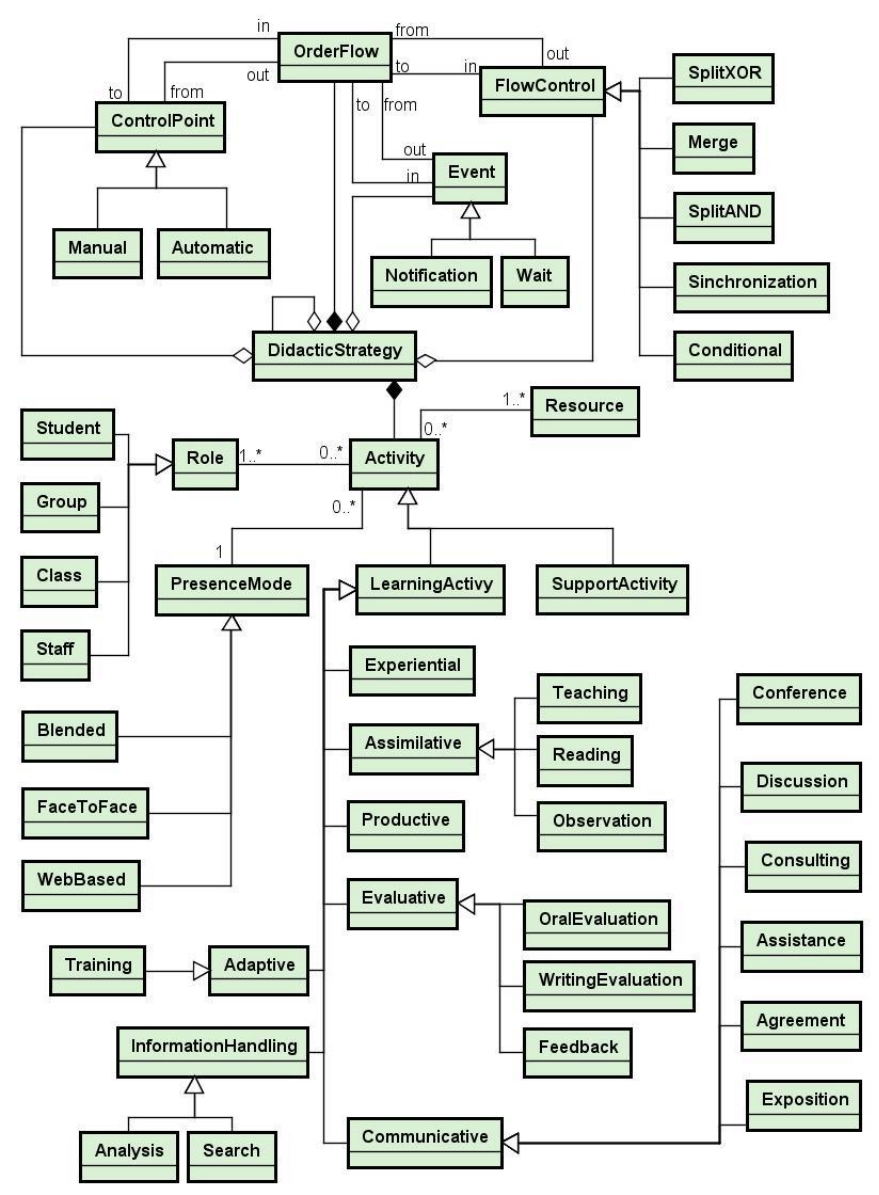

Figura 1. Modelo Conceptual de la estrategia didáctica (meta-modelo).

\section{MODELO CONCEPTUAL DE LA ESTRATEGIA DIDÁCTICA}

Teniendo en cuenta el modelo conceptual (meta-modelo o sintaxis abstracta) definido anteriormente, se desea crear un lenguaje visual que permita modelar estrategias didácticas. Los lenguajes notacionales definen su conjunto de reglas semánticas y sintácticas que establecen los elementos y las relaciones que son parte de los procesos. La especificación de la sintaxis concreta es uno de los pasos preliminares en la especificación de cualquier lenguaje, debido a que en ella se define sus particularidades y el mapping con respecto de la sintaxis abstracta. Esta especificación tiene como punto de inicio el análisis visual de cómo se presentarán al usuario los elementos de modelado del lenguaje. Este modelo define la estructura de componentes gráficos usados para representar los conceptos [36]. Diferentes estudios en la ingeniería de software confirman que la forma visual de la notación (sintaxis concreta) afecta significativamente el entendimiento, especialmente de las personas con poca experiencia (novatos). Por esta razón, y con el fin de dar cumplimiento a los requisitos $\mathbf{R 3}$ y $\mathbf{R 4}$, los investigadores decidieron construir la notación usando la teoría de PoN de D.L Moody [12]. La aplicación de esta teoría provee una base científica para la comparación, evaluación, mejora y construcción de notaciones visuales. La teoría PoN propone 9 principios que pueden ser usados exitosamente para construir lenguajes visuales de modelado gráfico. De manera resumida, los 9 principios se exponen en la Tabla II.

TABLA II

LOS 9 PRINCIPIOS PARA CONSTRUIR LENGUAJES VISUALES DE LA TEORÍA DE PON

\begin{tabular}{|c|c|}
\hline Principio & Definición \\
\hline 1. Ajuste Cognitivo & $\begin{array}{l}\text { use dialectos visuales diferentes cuando sea } \\
\text { necesario }\end{array}$ \\
\hline 2. Claridad Semiótica & $\begin{array}{l}\text { debe haber una correspondencia uno a uno } \\
\text { entre construcciones semánticas y símbolos } \\
\text { gráficos }\end{array}$ \\
\hline $\begin{array}{l}\text { 3. Discriminalidad } \\
\text { perceptual }\end{array}$ & $\begin{array}{l}\text { los símbolos deben ser claramente } \\
\text { distinguibles }\end{array}$ \\
\hline 4. Expresividad visual & $\begin{array}{l}\text { utilizar el rango completo y las capacidades } \\
\text { de las variables visuales }\end{array}$ \\
\hline $\begin{array}{l}\text { 5. Gestión de la } \\
\text { complejidad }\end{array}$ & $\begin{array}{l}\text { incluyen mecanismos para manejar la } \\
\text { complejidad }\end{array}$ \\
\hline 6. Integración Cognitiva & $\begin{array}{l}\text { incluye mecanismos explícitos para apoyar la } \\
\text { integración de información de diferentes } \\
\text { diagramas }\end{array}$ \\
\hline 7. $\mathrm{Tr}$ & use símbolos cuya apariencia evoque el \\
\hline Sem & $\begin{array}{l}\text { concepto que desea representar. Es } \\
\text { transparente si el símbolo informa su } \\
\text { significado, opaco si lo informa vagamente y } \\
\text { perverso si no lo informa o lo tergiversa. }\end{array}$ \\
\hline 8. Economía gráfica & $\begin{array}{l}\text { mantener el número de diferentes símbolos } \\
\text { gráficos cognitivamente manejable }\end{array}$ \\
\hline 9. Codificación dual & $\begin{array}{l}\text { enriquezca los diagramas con descripciones } \\
\text { textuales }\end{array}$ \\
\hline
\end{tabular}

La aplicación de estos principios se realizó de acuerdo con el proceso sistemático propuesto por Silva Teixeira [37], en donde los principios se aplican en 4 grupos: a) Principios básicos (claridad semiótica, transparencia semántica, y discriminalidad perceptual), b) Principios para manejar la complejidad (gestión de la complejidad, integración cognitiva) c) Principios de soporte (expresividad visual, economía gráfica y codificación dual) y d) Principio de conjunto de dialectos (ajuste cognitivo).

Los pasos que conforman el proceso sistemático son: 1) Definir el conjunto de dialecto (requisitos, objetivo, tamaño) aplicando el principio de conjunto de dialectos; 2) Diseñar el dialecto, en donde para cada constructor del modelo, a) se define un símbolo que los represente aplicando los principios básicos $\mathrm{y}, \mathrm{b})$ una vez definido el símbolo, se aplican los 
principios de soporte, repetir hasta que no haya más elementos a diseñar; e 3) Identificar formas de manejar la complejidad del modelo aplicando los principios para manejar la complejidad. A continuación, se presenta el análisis de cada principio de acuerdo al proceso descrito.

\section{A. Definición del conjunto de dialecto}

El proceso de diseño empieza con la identificación de requisitos que, en nuestro caso, se han establecido previamente en la sección II. Teniendo en cuenta el principio ajuste cognitivo, los autores establecieron que solo un dialecto es requerido para modelar la estrategia didáctica. El objetivo de este dialecto es proveer una notación visual simple e intuitiva para diseñar estrategias didácticas que permitan la especificación de unidades de aprendizaje. La notación debe contener símbolos para representar todos los constructores sin ambigüedad.

\section{B. Diseño del dialecto}

De acuerdo con el proceso sistemático, una vez definido el conjunto de dialecto se diseña cada símbolo que lo conforma. Para ello se aplican los principios básicos y una vez definidos todos los símbolos se aplican los principios de soporte. A continuación, se presenta el análisis para dichos principios:

(i) Principios Básicos: claridad semiótica, discriminalidad perceptual, transparencia semántica

Considerando la sintaxis abstracta definida por el metamodelo que se muestra en la iError! No se encuentra el origen de la referencia., y teniendo en cuenta el principio de claridad semiótica, una correspondencia 1:1 entre los constructores del meta-modelo y los símbolos gráficos fue definida. Este mapeo isomorfo tiene algunas excepciones: a) Los autores decidieron que no era necesario asignar un símbolo a los elementos FlowControl, PresenceMode, Role, ControlPoint, Activity, LearningActivity, Communicative, Assimilative, Evaluative, InformationHandling, Adaptive (elementos de modelado abstractos) sino solo a sus subtipos (concretos), debido a que los tipos abstractos no se instancian; y b) En el caso de los subtipos de actividad se decidió asignar el mismo símbolo (rectángulo) para los 18 subtipos (SupportActivity, Productive, Experiential, Teaching, Reading, Observation, Analysis, Search, Adaptive, OralEvaluation, WritingEvaluation, Feedback, Conference, Discussion, Consulting, Assitance, Agreement, Exposition). Esta decisión se tomó considerando el principio de discriminabilidad perceptual en donde los símbolos de una misma categoría tienen una pequeña distancia visual. Para diferenciarlos se utilizaron las variables visuales de color e icono. La decisión de usar el mismo símbolo también está soportada en que haber creado 18 símbolos diferentes podría afectar el principio de economía gráfica y por consiguiente el requisito R3. Concerniente a su transparencia semántica, el símbolo rectángulo asignado a cada subtipo de actividad es considerado semánticamente opaco debido a que no informa directamente su significado. Sin embargo, esto puede considerarse una buena decisión dado que este símbolo es fácil de recordar y está asociado con el símbolo "Actividad" de los diagramas de flujo utilizados por docentes en diferentes contextos.
Los iconos asignados a cada subtipo de actividad son semánticamente transparentes a excepción del icono de asesoría y evaluación oral que son opacos. Para llegar a esta afirmación se realizó un estudio de interpretación a ciegas, el cual se detalla en la sección 5 . El símbolo de actividad además de tener el icono que representa el tipo de actividad y la descripción se le asoció el tipo de rol y el modo de presencialidad de la actividad, ubicados en la parte superior izquierda del rectángulo. En el caso de los roles, los símbolos para Student, Group y Class son representados por una serie de círculos que, aunque son semánticamente opacos, pueden ser fáciles de recordar y con muy poco tiempo en el uso de la notación, los símbolos pueden cobrar el significado que les corresponde. Todos los roles tienen una pequeña distancia visual por estar asociados a una misma categoría. Igualmente, el modo de presencialidad es representado con el símbolo círculo. Para hacer distinción entre los diferentes modos se utilizó la variable texto. De esta manera los símbolos asociados a los roles y al modo de presencialidad tienen en cuenta el principio de discriminabilidad perceptual.

Los símbolos de control de flujo, Conditional, SplitAND, Sinchronization, corresponden a los utilizados en los diagramas de actividad de UML, los cuales son ampliamente usados en diferentes contextos y reportan altos niveles de efectividad cognitiva de acuerdo al estudio realizado en [38]. Igualmente, esta decisión se toma con base en la teoría del procesamiento de información gráfica de los humanos en donde, para ser entendido, la información de un diagrama debe ser integrada con conocimiento previo almacenado en la memoria a largo plazo [12]. El símbolo triangulo es asignado a SplitXOR y Merge. Se decidió asignar el mismo símbolo dado que se utilizan en conjunto, lo mismo que SplitAND y Sinchronization. Para diferenciarlos, se hizo una variación en la perspectiva de la forma (triangulo con punta hacia arriba para SplitXOR y triangulo con punta hacia abajo para Merge). Así, estos símbolos tienen una pequeña distancia visual teniendo en cuenta el principio de discriminalidad perceptual. La variable icono se adicionó para agregar significado que representara el comportamiento.

Para los puntos de control se asignó el símbolo circulo y se utilizó la variable icono para hacer diferencia entre un punto de control automatic y uno manual. La variable brillo (negro) se adicionó para darle relevancia visual con relación a su ubicación en un modelo de estrategia didáctica.

Finalmente, a los eventos se les asigno el símbolo hexágono y se utilizó la variable visual icono para hacer distinción entre notification y wait.

(ii) Principios de soporte: expresividad visual, economía gráfica y codificación dual

En relación con el principio de expresividad visual, la notación visual para modelar la estrategia didáctica usa como variable primaria la forma, la cual permite distinguir constructores semánticamente diferentes. De esta manera se tiene en cuenta la teoría de la primacía de la forma. Como variables secundarias usa el color, el brillo, la textura, la orientación y la posición. Por lo anterior, el lenguaje tiene una expresividad visual de 6 niveles debido a que usa 6 variables visuales para distinguir entre constructores semánticos. Las variables visuales que utilizan cada símbolo y su valor se 
presentan en la Tabla III. Por restricciones de espacio, las variables posición y orientación no se exponen en la tabla.

Como se observa en la Tabla III, los símbolos para los diferentes modos de presencialidad y los roles no tienen asignados formas convencionales debido a que se utilizan de forma integrada en el símbolo propio de la actividad. Así, se evita agregar mayor complejidad en la interpretación del modelo. Teniendo en cuenta la teoría de codificación redundante, cada símbolo utiliza diferentes variables visuales para incrementar la distancia visual entre uno y otro. Igualmente, cada símbolo tiene al menos un valor único sobre una variable visual de modo que sobresale (Pop up) en el campo visual sin esfuerzo consciente.

En relación con el principio de economía gráfica, $\mathrm{PoN}$ sugiere el uso de máximo 6 símbolos para representar categorías diferentes. Sin embargo, este número límite se aplica si solo se usa una variable visual. Al usar múltiples variables visuales para diferenciar entre símbolos puede incrementar la habilidad de discriminación humana casi de una manera aditiva. De este modo, el lenguaje propuesto al usar 6 variables visuales puede garantizar su expresividad visual para representar los 35 símbolos que lo conforman.

TABLA III

VARIABLES VISUALES PARA LOS SÍMBOLOS DEL LENGUAJE

\begin{tabular}{|c|c|c|c|c|c|}
\hline \multirow{2}{*}{ Símbolo } & \multicolumn{2}{|l|}{ Forma } & \multirow{2}{*}{ Brillo } & \multirow{2}{*}{ Color } & \multirow{2}{*}{ Textura } \\
\hline & Convencional & Icono & & & \\
\hline Start & Ovalo & $\mathrm{x}$ & Blanco & Verde & Solida \\
\hline End & Ovalo & $\mathrm{x}$ & Blanco & Rojo & Solida \\
\hline OrderFlow & Flecha & & Gris & & Solida \\
\hline Decision & Rombo & & Gris & & Solida \\
\hline SplitAND & Barra & & Gris & & Solida \\
\hline Sinchronization & Barra & & Gris & & Solida \\
\hline SplitXOR & Triangulo & $\mathrm{x}$ & Gris & & Solida \\
\hline Merge & Triangulo & $\mathrm{x}$ & Gris & & Solida \\
\hline Activity types & Rectángulo & $\mathrm{x}$ & & Varios & Degradado \\
\hline Role - Student & & $\mathrm{x}$ & Negro & & Solida \\
\hline Role - Group & & $\mathrm{x}$ & Negro & & Solida \\
\hline Role - Class & & $\mathrm{x}$ & Negro & & Solida \\
\hline Role - Staff & & $\mathrm{x}$ & Negro & & Solida \\
\hline Face-to-Face & & $\mathrm{x}$ & Negro & & Solida \\
\hline \multicolumn{6}{|l|}{ Mode } \\
\hline Blended Mode & & $\mathrm{x}$ & Negro & & Solida \\
\hline Web-based & & $\mathrm{x}$ & Negro & & Solida \\
\hline \multicolumn{6}{|l|}{ Mode } \\
\hline Automatic & Circulo & $\mathrm{x}$ & Negro & & Solida \\
\hline \multicolumn{6}{|l|}{ ControlPoint } \\
\hline Manual & Circulo & $\mathrm{x}$ & Negro & & Solida \\
\hline \multicolumn{6}{|l|}{ ControlPoint } \\
\hline Notification & Hexágono & $\mathrm{x}$ & & Rojo & Solida \\
\hline \multicolumn{6}{|l|}{ Event } \\
\hline Wait Event & Hexágono & $\mathrm{x}$ & & Rojo & Solida \\
\hline
\end{tabular}

Otras decisiones fueron tomadas con el fin de mantener el lenguaje lo más simple posible: a) Como se mencionó anteriormente, no se asignaron símbolos a las clases abstractas en el meta-modelo; y b) la información adicional a la actividad como la meta, los recursos que maneja, fecha de inicio y fin, entradas y salidas, aunque es información importante que debe ser considerada por el docente en el momento de la planificación de la estrategia didáctica, se dejó fuera del diagrama (off-diagram) para evitar excesiva complejidad gráfica y diagramática.
Finalmente, no se aplicó el principio Codificación Dual, que trata del uso del texto como complemento de información. Esto se debe a que, para los autores, la semántica de los símbolos que representan los constructores es lo suficientemente clara sin redundancia textual. Los autores consideran que en muy poco tiempo este tipo de información se hace irrelevante en el diagrama, dado que con el uso de los íconos es suficiente para su interpretación. Sin embargo, se tendrán en consideración el uso de etiquetas y texto alternativo para los iconos al momento de la implementación de la herramienta para el lenguaje, de tal manera que mitigue cualquier posible ambigüedad en su interpretación.

\section{Identificación de formas para manejar la complejidad}

Después de diseñar una versión inicial de la sintaxis concreta, es necesario evaluar si el lenguaje requiere de estrategias para manejar la complejidad del modelo. Los principios para manejar la complejidad (gestión de la complejidad e integración cognitiva) se enfocan en la importancia de manejar la complejidad diagramática, la cual es medida por el número de elementos en un diagrama, entre otros. En el caso del lenguaje DSML, los autores reconocen que los modelos de estrategias didácticas pueden tornarse muy extensos, lo cual podría afectar el entendimiento del modelo. En consecuencia, los autores decidieron introducir un símbolo para representar la relación que tiene consigo misma el constructor Didactic Strategy que encapsula un modelo de estrategia didáctica relacionado. Siguiendo el principio de discriminalidad perceptual, los diseñadores asignaron el símbolo carpeta y un icono, indicando que este elemento contiene otro modelo de estrategia didáctica.

\section{LENGUAJE PARA EL MODELADO DE LA ESTRATEGIA DIDÁCTICA}

Utilizando la notación definida en la sección IV y dando cumplimiento a los requisitos $\mathbf{R 2}$ y $\mathbf{R 5}$, nace el Lenguaje para el Modelado de la Estrategia Didáctica DSML. DSML es un lenguaje visual orientado al flujo y centrado en la actividad que permite el diseño del aprendizaje a través de estrategias didácticas que responden a necesidades específicas de los estudiantes. La representación de los diferentes tipos de actividad en el lenguaje permite identificar fácilmente patrones de enseñanza que puedan dar lugar a acciones de mejora o correctivas según sea el caso. En la Fig. 2 se presenta la sintaxis concreta resultante.

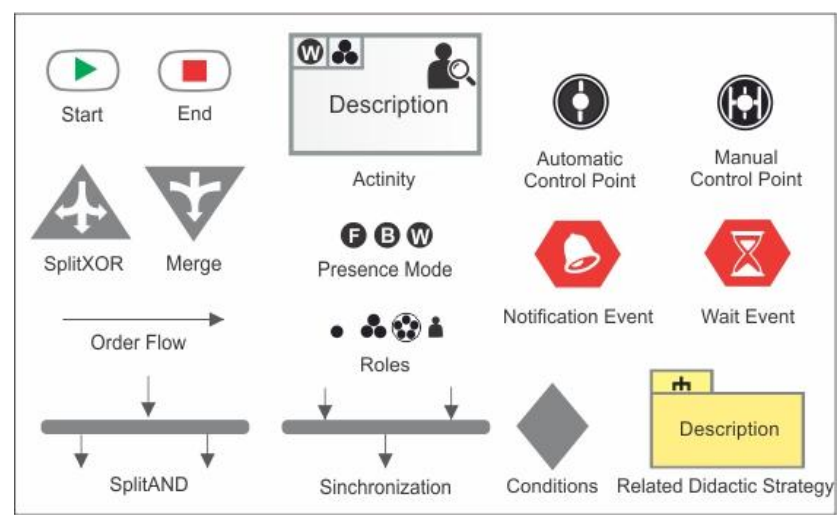


Figura 2. Notación visual para DSML.

A continuación, se define cada elemento de la notación:

(i) Start: Se utiliza para indicar el inicio de una estrategia didáctica.

(ii) End: Se utiliza para indicar el fin de una estrategia didáctica.

(iii) Conditional: Representa las comparaciones de dos o más valores. Tiene dos salidas de información, falso o verdadero.

(iv) SplitXOR: describe una situación donde varias actividades puedan ser iniciadas, pero solo una de ellas es seleccionada. La selección es decidida durante la ejecución.

(v) Merge (XOR): especifica el punto donde varias actividades que estaban siendo ejecutadas en paralelo convergen. Se evalúa que al menos una de las actividades que están asociadas al símbolo haya sido completada.

(vi) SplintAND: describe una situación donde varias actividades puedan ser iniciadas en paralelo.

(vii) Sinchronization: especifica el punto donde varias actividades que estaban siendo ejecutadas en paralelo convergen. Se evalúa que todas las actividades que están asociadas al símbolo hayan sido completadas.

(viii) OrderFlow: indica el seguimiento lógico del diagrama y el sentido de ejecución de las operaciones.

(ix) Learning Activity: indica una acción relacionada a una actividad didáctica.

(x) Presence Mode: describe el modo de presencialidad en el que ocurre la actividad, cuyos valores pueden ser "Face to face" (presencial), "Blended" (apoyado en TICs) y "Web-Based" (online).

(xi) Rol: describe el rol de quien realiza la actividad, los cuales pueden ser "Student" (estudiante), "Group" (grupo), "Class" (clase), "Staff" (personal a cargo).

(xii) Notificación: genera una alerta ya sea para el estudiante o para el docente (ej. Envío de un email cuando: se haya completado alguna tarea, una expresión se evalúa como verdadera o se fija alguna propiedad).

(xiii) Wait: establece un tiempo de espera (ej. Hasta una fecha determinada, o un número de horas o días a partir de su activación).

(xiv) Automatic / Manual ControlPoint: son puntos de control que estable el docente para evaluar el proceso de aprendizaje del estudiante hasta ese punto. La evaluación puede ser automatic, si la evaluación puede llevarse a la computación o manual si no es posible.

(xv) Related didactic strategy: encapsula un modelo de estrategia didáctica relacionado

El modelo de estrategia didáctica pretende, además de describir el proceso de aprendizaje del estudiante, ser una herramienta útil para el docente en la planificación de las actividades didácticas. Por esta razón, el símbolo de actividad, además de tener la descripción de la acción, tiene un color y un icono que refleja la naturaleza de la actividad de acuerdo a la taxonomía de Conole [11] y a los subtipos de actividad identificados, con ello, la semántica del diagrama se enriquece. En la Fig. 3 se presentan los iconos y el color asociado a los diferentes subtipos de actividades.

De esta manera, si un docente desea hacer una actividad de formación, DSML la denotará con el icono correspondiente y el color naranja. En la Fig. 4 se expone el meta-modelo para DSML y el símbolo asociado a cada constructor.

Finalmente, DSML se clasifica de acuerdo a los atributos del esquema de Botturi [19] como plano, formal, de implementación, simple y generativo o finalista, para las atributos estratificación, formalización, elaboración, perspectiva y creatividad, respectivamente.

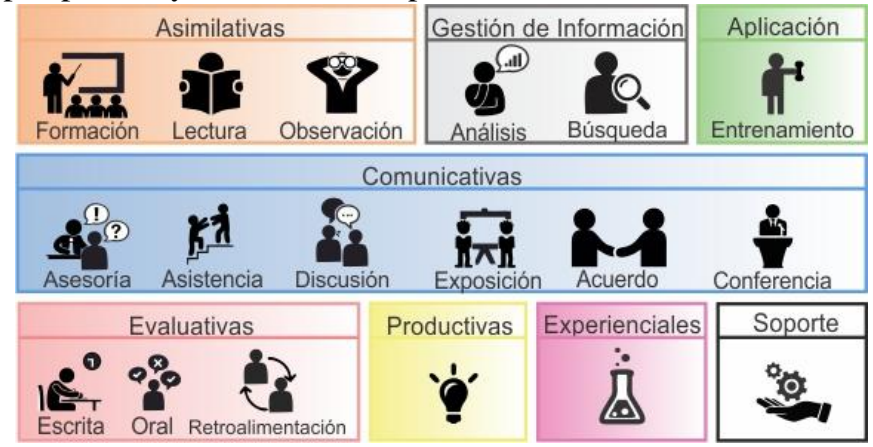

Figura 3. Color e iconografía para subtipos de actividad. 


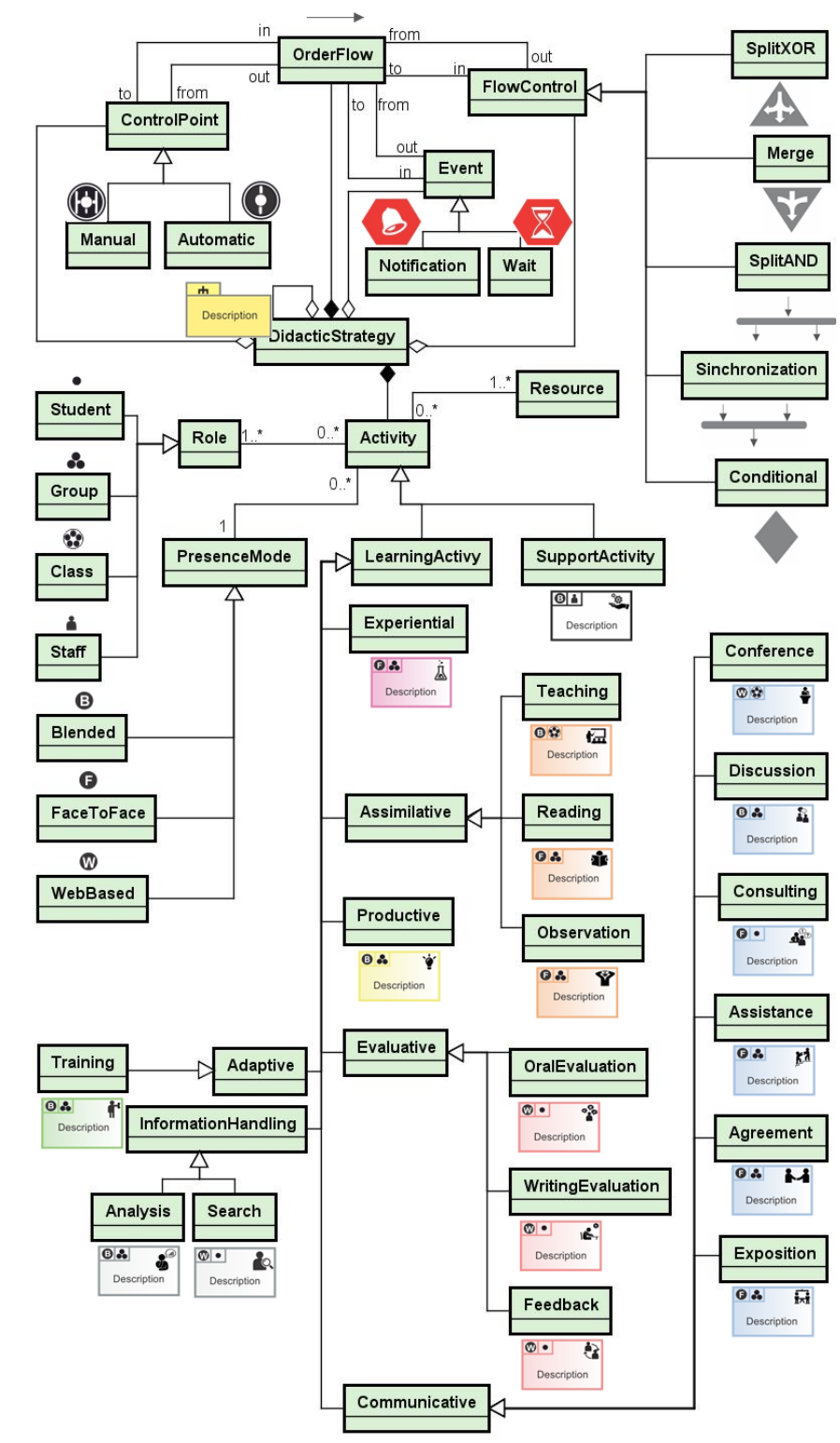

Figura 4. Símbolo asociado a los constructores del meta-modelo para DSML

\section{VALIDACIÓN DE LA ICONOGRAFÍA DEFINIDA PARA LAS ACTIVIDADES DE APRENDIZAJE DE DSML}

Una vez diseñado el lenguaje y teniendo en cuenta la importancia que tiene la clasificación de las actividades de acuerdo a la naturaleza de la tarea dentro de DSML, decidimos conducir un estudio que permitiera validar si los iconos seleccionados para representar cada actividad eran semánticamente transparentes. Para lograrlo, se realizó un estudio de interpretación a ciegas (también llamado prueba de comprensión [39] o prueba de reconocimiento [40]). Este tipo de prueba consiste en que los participantes deben inferir el significado de los símbolos que se les muestra. El nivel de comprensibilidad del símbolo es usualmente medido por el porcentaje de respuestas correctas. Sin embargo, los autores prefirieron usar el coeficiente de transparencia semántica [41], el cual mide la correlación entre la apariencia del símbolo y su significado y el resultado es más consistente con la definición teórica de transparencia semántica. Un coeficiente de transparencia semántica de un símbolo es calculado usando (1), formula basada en el concepto de análisis de Chicuadrados:

Frecuencia Máxima-Frecuencia Esperada

Total de respuestas-Frecuencia Esperada

La Frecuencia Esperada (número de respuestas esperadas al azar) $=n / s$, donde $n$ es el número de participantes en el grupo y $s$ es el número de símbolos. Si el concepto objetivo recibe el máximo número de respuestas, el coeficiente tendrá una señal positiva (transparente si es cercano a +1 y opaco si es cercano a 0), mientras que si un concepto distractor es el máximo, el valor tendrá una señal negativa (perverso).

Se realizó una prueba con 20 participantes que estuvieron divididos en dos grupos: el grupo 1 contaba con 10 docentes de la Universidad del Quindío (Colombia) y el grupo 2 con 10 estudiantes de doctorado de la Universidad Politécnica de Valencia (España), en su mayoría, estos últimos, con experiencia docente. Ambos grupos eran de diferentes áreas del conocimiento y no tenían ningún conocimiento previo de DSML. La prueba se realizó con 15 iconos de los 18 que conforman las actividades. La selección de los iconos para la prueba se realizó principalmente teniendo en cuenta los iconos de las actividades más comunes en el proceso de enseñanza. Inicialmente, los participantes del grupo 1 realizaron la prueba de interpretación a ciegas, en donde cada participante infirió un concepto que evocaba cada una de las imágenes que se les mostraba. Una vez terminaban la prueba se les pidió que realizaran un dibujo que diera a entender el concepto objetivo de los iconos en los que no habían acertado. Basados en los bosquejos y en los comentarios del grupo 1 se realizaron mejoras a los iconos y se aplicó nuevamente la prueba con el grupo 2. El coeficiente de transparencia semántica para cada uno de los iconos para ambos grupos se presenta en la Tabla IV.

Como se observa en la Tabla IV, los resultados para el Grupo 1 evidencian que los iconos para exposición, asesoría, análisis, acuerdo, conferencia, evaluación escrita y evaluación oral son semánticamente opacos (valores subrayados Grupo 1). De acuerdo con los cambios realizados a partir de las observaciones recibidas por el grupo 1, en la segunda parte del estudio, con el Grupo 2, se logró reducir la opacidad a solo dos iconos (valores subrayados Grupo 2), correspondientes a asesoría y evaluación oral. Igualmente, se logró aumentar el valor del coeficiente para los iconos que ya eran transparentes.

TABLA IV

RESULTADOS DEL COEFICIENTE DE TRANSPARENCIA SEMÁNTICA

\begin{tabular}{lcc}
\hline \multicolumn{1}{c}{ Icono } & Grupo 1 & Grupo 2 \\
\hline \hline Formación & 0,79 & 1,00 \\
Exposición & $\underline{0,25}$ & 0,79 \\
Discusión & 0,68 & 0,89 \\
Asesoría & $\underline{0,14}$ & $\underline{0,36}$ \\
Lectura & $\underline{1,00}$ & 1,00 \\
Observación & 0,89 & 0,89 \\
Análisis & $\underline{0,36}$ & 1,00 \\
Búsqueda & 0,79 & 1,00 \\
Entrenamiento & 0,89 & 0,79
\end{tabular}




\begin{tabular}{lll} 
Acuerdo & $\underline{0,25}$ & 0,57 \\
Conferencia & $\underline{0,36}$ & 1,00 \\
Asistencia & $\underline{0,79}$ & 1,00 \\
Evaluación Escrita & $\underline{0,25}$ & 0,57 \\
Evaluación Oral & $\underline{0,46}$ & $\underline{0,25}$ \\
Retroalimentación & 0,57 & 0,89 \\
\hline
\end{tabular}

\section{HERRAMIENTA DE AUTORÍA PARA DSML}

Desde el punto de vista de los autores, uno de los aspectos que dificulta la adopción de un EMLs, es la falta de herramientas de soporte amigables que simplifiquen el uso de estos lenguajes. El objetivo con la propuesta DSML es proveer una herramienta de autoría que pueda ser integrada a los LMS's de tal forma que se puedan crear estrategias didácticas que utilicen los recursos del LMS. Para lograr este objetivo, se propone aplicar el método expuesto en [14] que permite integrar el DSML con los LMS's de tal manera que el docente se centre en su labor en el momento de creación de la estrategia didáctica y no en el uso de TICs, además de otras ventajas. Para ilustrar esta integración con un LMS especifico, la Fig. 5 presenta una abstracción de la implementación de la herramienta de autoría DSML (meta-modelo en verde) con la plataforma Moodle (meta-modelo en azul). Con el fin de mantener la calidad de la imagen, solo se muestra una parte del meta-modelo DSML, el cual se expone completamente en la ¡Error! No se encuentra el origen de la referencia.

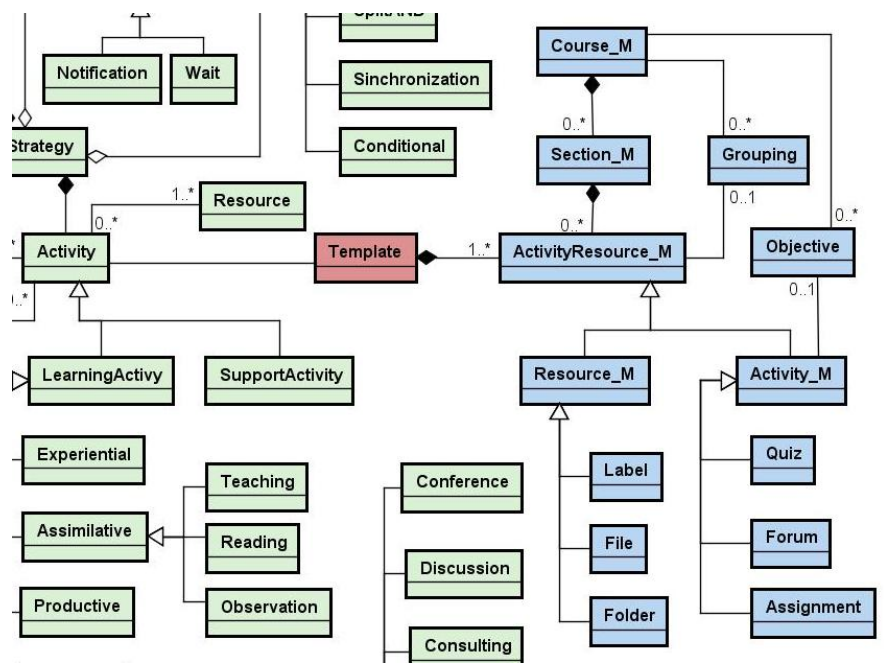

Figura 5. Modelo conceptual de implementación de la herramienta de autoría DSML en Moodle.

El meta-modelo de DSML y el meta-modelo del Moodle están relacionados entre sí a través del constructor Template (constructor rojo) que corresponde a un conjunto de plantillas (interfaces de usuario) diseñadas de acuerdo al método. En resumen, el método consiste en la clasificación de los componentes tecnológicos que provee un LMS en particular de acuerdo con las actividades de aprendizaje en las que pueden ser usados.

En este caso, los componentes tecnológicos de Moodle (en el diagrama ActivityResource_M) corresponden a chat, quiz, fórum, file...etc. Una vez clasificados los componentes, se obtiene una matriz en donde cada subtipo de actividad tiene asociado múltiples componentes. Posteriormente, se diseñan interfaces de usuario de acuerdo con las posibles variaciones de combinación de componentes especificadas en la matriz. Cada combinación debe responder a necesidades específicas de aprendizaje. Así, se crea un repositorio de plantillas, en donde cada plantilla (Template) está conformada por diferentes componentes tecnológicos (ActivityResource_M) y puede ser usada en diferentes actividades de aprendizaje (Activity). En [14], se realizó una prueba de concepto del método utilizando la plataforma Moodle y la notación de diagramas de flujo como EML. En este artículo, se presenta una versión mejorada de esta implementación utilizando como EML la notación DSML, para lo cual se crea una herramienta de autoría DSML independiente del LMS que posteriormente es integrada al Moodle. Una versión demo de la herramienta integrada al Moodle está disponible en URL: http://educationalmdl.byethost14.com/moodle30/login/index.p hp (usuario: admin, contraseña: Password1!)

La herramienta de autoría DSML se desarrolló con la librería javascript GoJS que facilita la creación de editores interactivos. La integración de la herramienta de autoría en el Moodle se realizó a través de la creación de un plugin que logra extender la navegación interna del Moodle, de modo tal que todos los cursos registrados en la plataforma disponen de un espacio de trabajo en donde se puede crear, modificar, visualizar e interactuar con la estrategia didáctica. Este plugin permite extender la base de datos de Moodle para poder consumir o escribir los datos correspondientes a la estrategia didáctica sin requerir de otras fuentes de datos externas a la plataforma. Los modelos son almacenados en la base de datos de Moodle y posteriormente renderizados dinámicamente a los estudiantes del curso dentro del espacio configurado para este fin. La Figura presenta la interfaz principal de la herramienta DSML integrada al Moodle. En esta interfaz, el profesor construye el modelo de estrategia didáctica arrastrando los diferentes símbolos a la zona de trabajo de acuerdo con los tipos de actividades que desea realizar, a los objetivos de aprendizaje y a posibles estilos de aprendizaje que haya detectado en sus estudiantes. Cada elemento del modelo tiene asociados ciertos atributos que se editan en la zona de propiedades. En el caso de una actividad de aprendizaje, el profesor deberá introducir el nombre, tipo, subtipo, actor, modo de presencialidad, fecha inicio y cierre $\mathrm{y}$ retroalimentación. Adicional a estos atributos, el profesor debe seleccionar la plantilla que desea utilizar para la actividad en particular y relacionar los recursos de acuerdo con la selección. Una vez configurado todo el modelo, en la vista del estudiante aparecerán las actividades a realizar, de acuerdo con el orden definido en el modelo.

Como prevalidación del lenguaje y de la herramienta de autoría se modeló la estrategia didáctica correspondiente al curso de desarrollo dirigido por modelos impartido en la Universidad Politécnica de Valencia. El curso se encuentra estructurado en 6 módulos, en donde sus principales actividades de aprendizaje son de formación, lectura, entrenamiento, evaluación y productiva. Las actividades de entrenamiento están relacionadas a ejercicios que van 
aumentando en su complejidad de uno módulo a otro y la productiva es un proyecto en donde el estudiante debe aplicar todos los conocimientos adquiridos. Este proyecto puede ser desarrollado en paralelo a medida que van avanzando en los módulos o al final del curso. En la Fig. 6 se observa una porción de la estrategia didáctica correspondiente al curso.

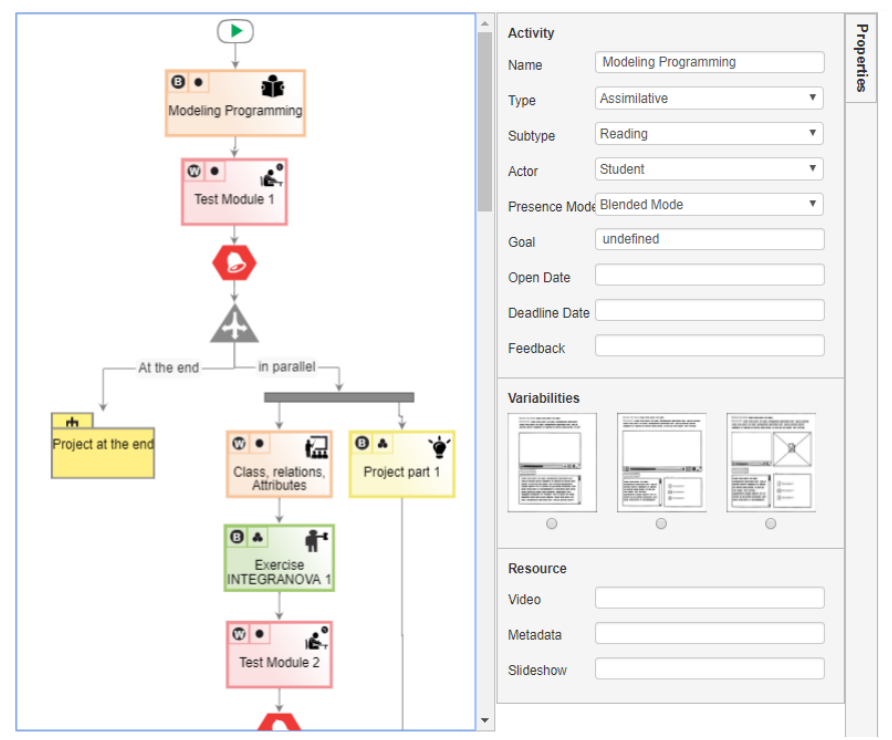

Figura 6. Herramienta de autoría DSML en la plataforma Moodle

\section{CONCLUSIONES Y TRABAJOS FUTUROS}

Este artículo presenta el diseño del lenguaje para el modelado de estrategias didácticas DSML, un lenguaje visual, orientado a la actividad para el diseño del aprendizaje. La característica relevante del lenguaje es la representación de las diferentes actividades de acuerdo con la naturaleza de la tarea, lo cual permite identificar fácilmente patrones de enseñanza que pueda dar a lugar a acciones de mejora o correctivas según sea el caso. Los requisitos del lenguaje se establecieron a partir de la exploración de fortalezas y debilidades de propuestas EML existentes. Establecidos los requisitos, el lenguaje se diseña de acuerdo con los principios de PoN bajo el proceso sistemático expuesto por da Silva Teixeira [37]. También se presenta la herramienta de autoría para DSML, la cual es integrada a un LMS utilizando el método expuesto en [14]. El estudio de interpretación a ciegas permitió validar el principio de transparencia semántica de la iconografía de las actividades. Como prevalidación de todo el lenguaje, se diseñó un curso de Model Driven.

Gracias al proceso que se detalla en este artículo se ha dado cumplimiento parcial a los requisitos establecido inicialmente. Así, DSML es un lenguaje que parte del contexto educativo (R1 sección III), que permite diseñar el aprendizaje a través de la especificación de la actividad (que hacer, por quien y con qué, $R 2$ sección $V$ ), que se estima ser fácil de usar y de aprender debido a que es un lenguaje visual diseñado aplicando los principios de efectividad cognitiva $(R 3, R 4, R 5$ sección $I V$ y $V I)$ y que puede ser llevado a la computación ( $R 6$ sección VII).
Con el fin de dar cumplimiento total a los requisitos $\mathrm{R} 3 \mathrm{y}$ R4, se deben realizar otros estudios. Por ejemplo, estudios empíricos de usabilidad como los expuestos en [42-44] puede ser llevados a cabo para determinar si el lenguaje es fácil de usar y de aprender. Igualmente, como no existen métricas para todos los principios de PoN, estudios empíricos por comparación como los realizados en [38, 45, 46], podrían determinar el nivel de efectividad cognitiva de la notación propuesta.

Finalmente, futuras investigaciones son requeridas para aprovechar en su totalidad las bondades de la integración de DSML con un LMS a saber: 1) adoptar un mecanismo estándar para el almacenamiento de modelos de tal manera que se puedan compartir y reutilizar modelos de estrategia didáctica ya creados, y 2) crear un editor y generador de interfaces para los diferentes tipos de actividades, con el fin de ampliar las posibilidades de presentación de acuerdo a los propósitos de la actividad y a los recursos disponibles.

\section{REFERENCES}

[1] P. Marqués. (2011). La Enseñanza Buenas Prácticas. La Motivación. Available: http://peremarques.net/

[2] G. R. Morrison, S. M. Ross, J. E. Kemp, and H. Kalman, Designing Effective Instruction: John Wiley \& Sons, 2010.

[3] R. Koper, "Modeling units of study from a pedagogical perspective The pedagogical metamodel behind EML," ed. Open University of the Netherlands, 2001.

[4] R. Koper, B. Olivier, and T. Anderson, "IMS Learning Design Information Model," ed: IMS Global Learning Consortium, 2003.

[5] M. Caeiro, M. Llamas, and L. Anido, "Un Lenguaje Gráfico para el Modelado de Unidades Didácticas en Ingeniería," IEEE-RITA, vol. 2, 2007.

[6] D. Michael and M.-P. Renate, "coUML: A Visual Language for Modeling Cooperative Environments," in Handbook of Visual Languages for Instructional Design: Theories and Practices, B. Luca and S. Todd, Eds., ed Hershey, PA, USA: IGI Global, 2008, pp. 154182.

[7] L. Botturi, "E2ML: A Visual Language for the Design of Instruction," Educational Technology Research and Development, vol. 54, pp. 265293, 2006.

[8] J. Bundsgaard, "Introducing the Collaborative Learning Modeling Language (ColeML)," in Proceedings of the 13th Conference on Elearning, ECEL 2014, 2014, pp. 81--88.

[9] G. Paquette, F. Crevier, C. Aubin, J. Rocheleau, C. Paquin, and M. Léonard, "Méthode d'ingénierie d'un système d'apprentissage (MISA)," Revue Informations In Cognito, vol. 8, 1997.

[10] G. Paquette, "Educational Modeling Languages, from an Instructional Engineering Perspective. ," in Online education using learning objects ed London: R. McGreal., 2004, pp. 331-246. .

[11] G. Conole, "Describing learning activities: Tools and resources to guide practice," in Rethinking Pedagogy for a Digital Age: Designing and Delivering E-learning, H. Beetham and R. Sharpe, Eds., ed: Routledge, 2007.

[12] D. Moody, "The Physics of Notations: Toward a Scientific Basis for Constructing Visual Notations in Software Engineering," IEEE Transactions on Software Engineering, vol. 35, pp. 756-779, 2009.

[13] D. S. Saito and V. R. Ulbricht, "Learning Managent Systems and Faceto-Face Teaching in Bilingual Modality (Libras/Portuguese)," IEEE Latin America Transactions, vol. 10, pp. 2168-2174, 2012.

[14] A. Ruiz, W. J. Giraldo, and J. Arciniegas, "Method for the Integration of the Didactic Strategy Model in Virtual Learning Platforms in the University Context: A mechanism that takes into account the professor's work " in 12 Congreso Colombiano de Computación 12CCC, Cali - Colombia, 2017.

[15] I. Martínez-Ortiz, P. Moreno-Ger, J. L. Sierra-Rodríguez, and B. Fernández-Manjón, "A Flow-Oriented Visual Language for Learning Designs," in Advances in Web Based Learning - ICWL 2008: 7th International Conference, Jinhua, China, August 20-22, 2008. 
Proceedings, F. Li, J. Zhao, T. K. Shih, R. Lau, Q. Li, and D. McLeod, Eds., ed Berlin, Heidelberg: Springer Berlin Heidelberg, 2008, pp. 486496.

[16] L. Botturi, "E2ML - Educational Environment Modeling Language " Educational Technology Research and Development, vol. 54, pp. 265293,2006

[17] L. Botturi, "Visual Languages for Instructional Design: an Evaluation of E2ML," presented at the EdMedia: World Conference on Educational Media and Technology 2004, Lugano, Switzerland, 2004.

[18] I. Global. (2003). IMS Learning Design Specification Available: http://www.imsglobal.org/learningdesign/

[19] L. Botturi, M. Derntl, E. Boot, and K. Figl, "A Classification Framework for Educational Modeling Languages in Instructional Design," presented at the IEEE International Conference on Advanced Learning Technologies (ICALT 2006), Kerkrade, The Netherlands, 2006.

[20] R. R. Amorim, M. Lama, E. Sánchez, A. Riera, and X. A. Vila, "A Learning Design Ontology based on the IMS Specification," Educational Technology \& Society, vol. 9, pp. 38-57, 2006.

[21] L. M. Serrano-Cámara, M. Paredes-Velasco, A. Ahijado-Sánchez, and J. Á. Velázquez-Iturbide, "Modeling the Collaborative Instructional Framework for LMSs Using Educational Modeling Languages," IEEE Revista Iberoamericana de Tecnologias del Aprendizaje, vol. 10, pp. 43-50, 2015.

[22] M. Caeiro, M. Llamas, and L. Anido, "PoEML: Modeling learning units through perspectives," Computer Standards \& Interfaces, vol. 36, pp. 380-396, 2014/02/01/ 2014.

[23] J. M. Dodero, Á. M. del Val, and J. Torres, "An extensible approach to visually editing adaptive learning activities and designs based on services," Journal of Visual Languages \& Computing, vol. 21, pp. 332$346,2010 / 12 / 20 / 2010$.

[24] A. Berggren, D. Burgos, J. M. Fontana, D. Hinkelman, V. Hung, A. Hursh, and G. Tielemans, "Practical and Pedagogical Issues for Teacher Adoption of IMS Learning Design Standards in Moodle LMS. ," Journal of Interactive Media in Education, 2005.

[25] K. Figl, M. Derntl, M. C. Rodriguez, and L. Botturi, "Cognitive effectiveness of visual instructional design languages," Journal of Visual Languages \& Computing, vol. 21, pp. 359-373, 2010/12/20/ 2010.

[26] L. Botturi, D. Burgos, M. Caeiro, M. Derntl, R. Koper, P. Parrish, T. Sodhi, and C. Tattersall, "Comparing Visual Instructional Design Languages: A Case Study.," in Handbook of Visual Langauges in Instructional Design: Theories and Pratices, L. B. T. Stubbs, Ed., ed Hershey: IGI Global 2008, pp. 315-343.

[27] M. Derntl and R. Motschning-Pitrik, "coUML - A Visual Language for Modeling Cooperative Environments," in Instructional Design: Concepts, Methodologies, Tools and Applications: Concepts, Methodologies, Tools and Applications, I. R. Management Association, Ed., ed: Information Science Reference, 2011.

[28] H. D. Jørgensen. (2004). Interactive process models.

[29] G. Meneses, "NTIC, Interacción y Aprendizaje en la Universidad," Doctorado, Departament de Pedagogia, UNIVERSITAT ROVIRA I VIRGILI, 2007.

[30] S. Torre, "La comunicación didáctica: modelos y pautas para la acción," in Didáctica general para psicopedagogos / coord. por Núria Rajadell i Puiggròs, Félix Sepúlveda, , ed, 2001, pp. 103-154.

[31] J. L. Rodríguez, "Curriculum, acto didáctico y teoría del texto," Anaya, Ed., ed España, 1985.

[32] A. González, "Enseñanza, profesores y universidad.," ed. Tarragona: Universitat Rovira i Virgili. Institut de Ciències de l'Educació. , 2002.

[33] S. J. Gimeno, Teoría de la Enseñanza y desarrollo del curriculo. , Cuarta Edición ed. Buenos Aires. : Rei Argentina, 1992.

[34] A. Ferrandez, Didàctica i components de l'acte didàctic. : EDIOUC Edicions de la Universitat Oberta de Catalunya, 2002.

[35] C. Marcelo, C. Yot, C. Mayor, M. Sánchez, P. Murillo, J. M. Rodríguez, and A. Pardo, "Las actividades de aprendizaje en la enseñanza universitaria: ¿hacia un aprendizaje autónomo de los alumnos?," Revista de Educación, p. 363, 2014.

[36] W. J. G. Orozco, "Marco de Desarrollo de Sistemas Groupware Interactivos Basado en la Integración de Procesos y Notaciones," Escuela Superior de Informática de Ciudad Real, 2010.

[37] M. d. G. da Silva Teixeira, G. K. Quirino, F. Gailly, R. de Almeida Falbo, G. Guizzardi, and M. Perini Barcellos, "PoN-S: A Systematic Approach for Applying the Physics of Notation (PoN)," in Enterprise, Business-Process and Information Systems Modeling: 17th
International Conference, BPMDS 2016, 21st International Conference, EMMSAD 2016, Held at CAiSE 2016, Ljubljana, Slovenia, June 13-14,2016, Proceedings, R. Schmidt, W. Guédria, I. Bider, and S. Guerreiro, Eds., ed Cham: Springer International Publishing, 2016, pp. 432-447.

[38] K. Figl, J. Mendling, and M. Strembeck, "Towards a usability assessment of process modeling languages," in Proc. of the 8th Workshop Geschäftsprozessmanagement mit Ereignisgesteuerten Prozessketten (EPK 2009), Berlin, Germany. CEUR Workshop Proceedings, 2009, pp. 118-136.

[39] J. J. Foster, "Graphical Symbols: Test Methods for Judged Comprehensibility and for Comprehension.," ed: ISO Bulletin, 2001, pp. 11-13.

[40] W. C. Howell and A. H. Fuchs, "Population stereotypy in code design," Organizational Behavior and Human Performance, vol. 3, pp. 310-339, 1968/08/01/ 1968.

[41] P. Caire, N. Genon, P. Heymans, and D. L. Moody, "Visual notation design 2.0: Towards user comprehensible requirements engineering notations," in 2013 21st IEEE International Requirements Engineering Conference (RE), 2013, pp. 115-124.

[42] D. Albuquerque, B. Cafeo, A. Garcia, S. Barbosa, S. Abrahão, and A. Ribeiro, "Quantifying usability of domain-specific languages: An empirical study on software maintenance," Journal of Systems and Software, vol. 101, pp. 245-259, 2015/03/01/2015.

[43] Ewais Ahmed and O. De Troyer, "A Usability Evaluation of Graphical Modelling Languages for Authoring Adaptive 3D Virtual Learning Environments," in Proceedings of the 6th International Conference on Computer Supported Education (CSEDU 2014), , Barcelona, Spain, 2014.

[44] C. Kossow, T. Helms, J. M. Kreutzer, A. Martens, and A. M. Uhrmacher, "Evaluating different modeling languages based on a user study," presented at the Proceedings of the 49th Annual Simulation Symposium, Pasadena, California, 2016.

[45] M. Saeed, F. Saleh, S. Al-Insaif, and M. El-Attar, "Evaluating the Cognitive Effectiveness of the Visual Syntax of Feature Diagrams," in Requirements Engineering: First Asia Pacific Requirements Engineering Symposium, APRES 2014, Auckland, New Zealand, April 28-29, 2014. Proceedings, D. Zowghi and Z. Jin, Eds., ed Berlin, Heidelberg: Springer Berlin Heidelberg, 2014, pp. 180-194.

[46] M. Saeed, F. Saleh, S. Al-Insaif, and M. El-Attar, "Empirical validating the cognitive effectiveness of a new feature diagrams visual syntax," Information and Software Technology, vol. 71, pp. 1-26, 2016/03/01/ 2016.

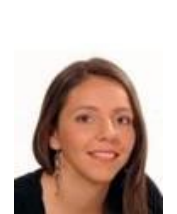

A. Ruiz was born in Colombia in 1979. She recived her degree in system engineer from Universidad del Quindío in 2002 and ecommerce master degree from Instituto Tecnológico de Monterrey in 2008. Currently, she is doing her $\mathrm{PhD}$ in telematics engineer at Universidad del Cauca and she is full professor at the Universidad del Quindío. She works software engineering, databases, usability, human computer interaction, interactive television and ecommerce. She is focused on methodologies and frameworks for developing interactive system based on video content distribution and usability throughout her $\mathrm{PhD}$ thesis. She is researcher of SINFOCI Research Group (Grupo de Investigación en Sistemas de Información y Control Industrial) of Universidad del Quindío Mrs Ruiz is member of the usability Lab in Universidad del Quindío, Vivelab Quindío and AIPO.

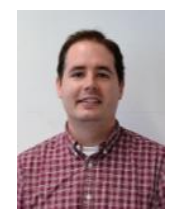

J. I. Panach is assistant professor at the Universitat de València and researcher at the PROS Research Center (Centro de Investigacion en Metodos de Produccion de Software) of the Universidad Politecnica de Valencia. Ignacio got his Ph. D in Computer Science in May 2010. His research activities focus on the model-driven development context. Currently working on how to model usability at the first stages of the software development process, how to capture usability requirements and how to evaluate automatically the system usability from conceptual models. He has more than 30 research papers in conferences, journals and books.

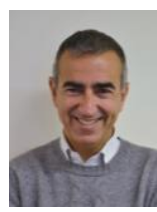

O. Pastor is Full Professor and Director of the "Centro de Investigación en Métodos de Producción de Software (PROS)" at the Universidad Politécnica de Valencia (Spain). He received his Ph.D. in 1992. He was a researcher at HP 
Labs, Bristol, UK. Supervisor of 20 completed PhD theses and 31 completed Masters theses on topics that relate to Conceptual Modeling, he has published more than three hundred research papers in conference proceedings, journals and books, received numerous research grants from public institutions and private industry, and been keynote speaker at several conferences and workshops. Chair of the ER Steering Committee (2009-10), ER Fellow since 2010, member of the SC of conferences as CAiSE, ER, ICWE, ESEM, CIbSE or RCIS, and member of over 100 Scientific Committes of top-ranked international conferences, his research activities focus on conceptual modeling, web engineering, requirements engineering, information systems, and model-based software production. He created the object-oriented, formal specification language OASIS and the corresponding software production method OO-METHOD. He led the research and development underlying CARE Technologies that was formed in 1996. CARE Technologies has created an advanced MDA-based Conceptual Model Compiler called Integranova, a tool that produces a final software product starting from a conceptual schema that represents system requirements. He is currently leading a multidisciplinary project linking Information Systems and Bioinformatics notions, oriented to designing and implementing tools for Conceptual Modeling-based interpretation of the Human Genome information

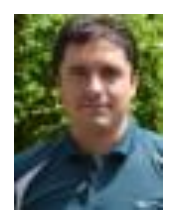

F. D. Giraldo recived his degree in system engineer from Universidad del Quindío in 2004 and informatics master degree from Universidad EAFIT in 2011. He is finishing PhD in computing from Universidad Politécnica de Valencia (Spain). He is researcher of SINFOCI Research Group (Grupo de Investigación en Sistemas de Información y Control Industrial) of Universidad del Quindío. He is member of PROS Research Center (Centro de Investigación en Métodos de Producción Software) of the Universidad Politécnica de Valencia (Spain). He is full professor at the Universidad del Quindío.

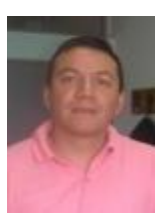

J. L. Arciniegas is Electronics and Telecommunications engineer, Network and Telematic Services Specialist, he wrote his doctoral thesis on Quality-driven Evolutionary Software Development process for Service Oriented Architectures at the Department of Telematics, Universidad Politécnica de Madrid, Spain. Currently he is full professor in the Universidad del Cauca. He works with software architecture, quality of software, architecture recovery, software process improvement and interactive multimedia services. In particular, he has focused in the last eight years on IPTV and interactive multimedia services through a number of related projects funded by Colciencias, Colombian Educational Ministry, CYTED, LACCIR and other sources. He is co-author of several research papers.

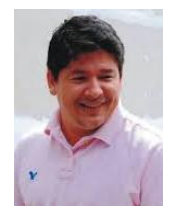

W. J. Giraldo is Electrical engineer, automatic master and advanced computer master, he wrote his doctoral thesis on Development Framework of groupware interactive system based on integration of notation and process CIAF, Universidad Castilla-La Mancha, Spain. Currently he is full professor in the Universidad del Quindío. He is interested on usability, Human Computer Interaction, Model driven, development methodologies, software engineering, and groupware interactive systems. He has focused in the last ten years on promoting $\mathrm{HCI}$ in Colombia. $\mathrm{He}$ is co-author of several research papers. 